\title{
Real Time Implementation of CAP Modulation with 'Better-than-Nyquist' Pulse Shaping in Visible Light Communications
}

\author{
Paul Anthony Haigh and Izzat Darwazeh
}

\begin{abstract}
In this paper we experimentally verify a realtime implementation of the 'better-than-Nyquist' pulse shape in carrier-less amplitude and phase (CAP) modulation in the context of a visible light communications system. We use two National Instruments universal software radio peripherals (USRPs) as a transmitter and receiver working independently. Previously reported experimental work on pulse shaping in CAP is also verified in real time, showing lower error vector magnitudes (EVM) can be obtained at shorter filter lengths using the betterthan-Nyquist pulses in place of the conventionally used squareroot raised cosine as the basis function of the signal. We show that a real time EVM penalty is an additional $3 \% \mathrm{EVM}$ in the worst case and is a result of synchronisation offset at the receiver.
\end{abstract}

\section{INTRODUCTION}

As visible light communications (VLC) systems become increasingly popular, advanced modulation formats and digital signal processing techniques have become two of the main choices to improve transmission speeds. In terms of advanced modulation formats, orthogonal frequency division multiplexing (OFDM) and carrier-less amplitude and phase (CAP) modulation and their derivatives have been the most commonly deployed within VLC systems [1-3]. In particular, it was reported in [1] that CAP outperforms OFDM in terms of bit-error rate (BER), achieving a data rate of $\sim 3.22 \mathrm{~Gb} / \mathrm{s}$ in comparison to $\sim 2.93 \mathrm{~Gb} / \mathrm{s}$ at a lower BER. The additional performance is an advantage however comes at the cost of increased computational complexity; CAP modulation typically requires at least four finite impulse response (FIR) filters with a high number of taps, which increases their complexity beyond the (inverse) fast Fourier transform (I)FFT paid required in OFDM [4]. The computation complexity of the (I)FFT pair increases with $2 \log _{2}(N)$, where $N$ is the number of subcarriers while the FIR filters increase with $4 L_{s}$, where $L_{s}$ is the number of taps [4]. Therefore, when either the number of samples-per-symbol $\left(n_{s s}\right)$ or the filter symbol span $(Z)$ are reasonably increased, since $L_{s}=Z n_{s s}+1$, CAP becomes more computationally complex than OFDM, disadvantageously. The sampling frequency of the signal is set to $f_{s}=R_{s} n_{s s}=4 \mathrm{MHz}$.

This work was supported by the UK EPSRC grant EP/P006280/1: Multifunctional Polymer Light-Emitting Diodes with Visible Light Communications (MARVEL).

PAH is with the Intelligent Sensing and Communications Group, Newcastle Unviersity, NE1 7RU, UK. email: paul.haigh@ newcastle.ac.uk

$\mathrm{PAH}$ was with and ID is with the Information and Communications Engineering Group, University College London, Gower Street, WC1E 6BT, United Kingdom e-mail: i.darwazeh@ucl.ac.uk
In recent work $[4,5]$, it has been shown that the number of FIR filters required in CAP can be reduced by $50 \%$, since the transmitter filter pair can be abstracted to look-up tables once their coefficients have been predetermined. Typically, CAP modulation is based on the formation of a Hilbert pair of pulse shaping filters that occupy the same frequencies but are separated in phase by $\pi / 2$ rads. These filters are generated as the product of a basis function and cosine/sine pair, where the basis function is typically a square-root raised cosine (SRRC), and the cosine/sine is generated at the carrier frequency of the signal [6]. This remained unchanged since the conception of CAP modulation in [7] until recently, when reports demonstrated that full-Nyquist pulses can be used as the basis function in combination with quadrature amplitude modulation (QAM) receivers [4], i.e. a local oscillator and a low-pass filter instead of time-reversed matched filters. It was also shown in the literature that overall complexity can be reduced by $\sim 90 \%$, at a signal-to-noise ratio (SNR) penalty of $\sim 2 \mathrm{~dB}$ compared to the conventional method [6].

One such example of a full-Nyquist pulse shape is the 'better-than-Nyquist' (BTN) pulse, which has several advantages over the traditional SRRC that is used in CAP systems. The main advantage of the BTN pulse is that it dampens faster than the SRRC and therefore less energy is contained in the side-lobes. This means that it can tolerate lower filter spans and fewer filter taps, which is advantageous as the BER of CAP systems has demonstrated a strong dependence on the filter length [8]. In [9], it was shown that using the BTN pulse as the basis function with QAM receivers significantly alleviates this dependence, with similar BERs shown for both a low and high number of filter taps.

In the literature in recent years, there have been very few demonstrations of real time (RT) operation of VLC systems $[10,11]$, both of which are based on OFDM in spite of the aforementioned advantages of CAP modulation. In this work, we expand on the reports in [6,9] by developing and experimentally demonstrating a CAP system in RT. In terms of CAP, there have been two reports in the literature $[12,13]$, both of which do not test basis functions beyond the SRRC. Thus, we experimentally test our RT system to verify the BER results shown in [9] in RT, with a slight penalty related to the synchronisation of the signal.

\section{Real Time Test Setup}

The experimental system is illustrated via a block diagram in Fig. 1. A pseudorandom binary sequence $\mathbf{D}_{\mathbf{s}}$ of length 


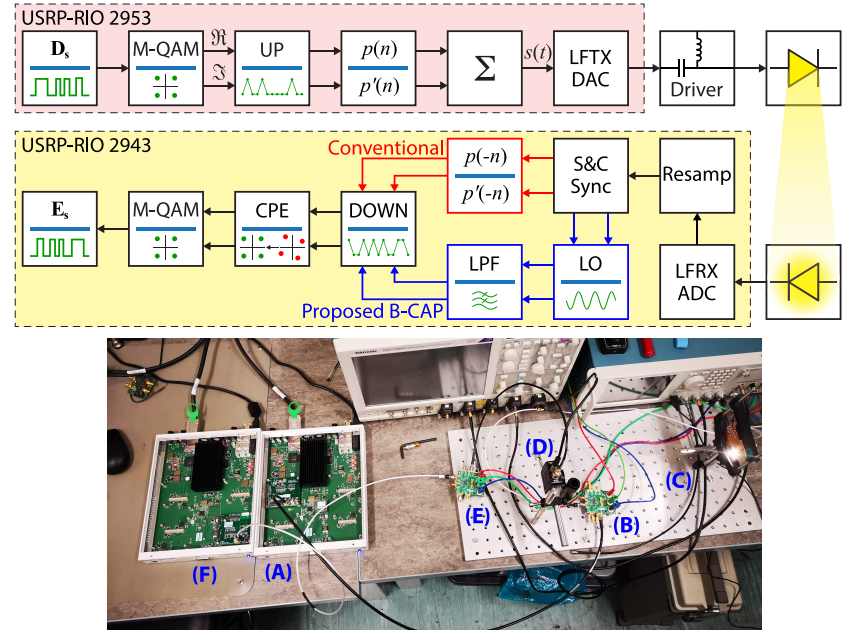

Fig. 1. Simplified block diagram of the proposed system and photograph of the link. Index A: USRP-RIO2953, B: TH3202, C: LED, D: PD, E: TH3202 and F: USRP-RIO 2943

$2^{13}-1$ is generated and mapped into symbols using the $M$-ary quadrature amplitude modulation ( $M$-QAM) constellation, where $M=2^{k}$ and $k$ is set sequentially to 2,4 and 6 bits/symbol. The data is then up-sampled at a rate of $n_{s s}=\mu\lceil 2(1+\beta)\rceil$ which includes an oversampling rate $\mu$ of 2 and $\beta$ is the roll-off factor of the basis function used to form the pulse shaping filters. The pulse shaping filters are generated as the product of a basis function $g(t)$ with a cosine and sine wave which define the band-limitations of the signal. The frequencies of the cosine and sine waves used to generate the in-phase $p(t)$ and quadrature $p^{\prime}(t)$ components of the Hilbert pair are set to the symbol rate. The symbol rate $R_{s}$ is given as $R_{S}=B /(1+\beta)$. The bandwidth $B$ is set to $1 \mathrm{MHz}$ and $\beta=0.1$ to maintain consistency with [9]. The basis function has traditionally been an SRRC as mentioned, while in [9] we experimentally demonstrated that advantages in BER performance could be gained by replacing it with the BTN pulse. Therefore, we test both of these in real-time. The SRRC basis function is given by [9]:

$$
g_{S}(t)=\frac{2 \beta\left[\cos ((1+\beta) \xi)+\sin ((1-\beta) \xi) \gamma^{-1}\right]}{\pi \sqrt{T_{S}}\left[1-\gamma^{2}\right]}
$$

where $T_{s}=1 / R_{s}$ is the symbol period, $\gamma=4 \beta t T_{s}^{-1}, \xi=\pi t T_{s}^{-1}$ and $t$ is the instantaneous time sample. The BTN basis function is given by [9]:

$$
g_{B}(t)=\frac{\sin (\xi)\left[\xi \frac{2 \beta}{\ln (2)} \sin (\beta \xi)+\cos (\beta \xi)-1\right]}{\xi\left[\left(\xi \frac{2 \beta}{\ln (2)}\right)^{2}+1\right]}
$$

The pulse shapes are given by [9]:

$$
\begin{aligned}
& p(t)=g(t) \cos \left(2 \pi f_{c} t\right) \\
& p^{\prime}(t)=g(t) \sin \left(2 \pi f_{c} t\right)
\end{aligned}
$$

where $g(t)$ is either the SRRC eq.(1) or BTN eq.(2) basis function, both are shown in Fig. 2. Clearly, the BTN pulse has less energy in the side-lobes which will be advantageous later, since it means it can be realised with shorter filter span.
The pulse shaping filters are realised in look-up table form to reduce complexity, and hence, they are pre-determined based on the value of $\beta$ and the filter length. The filter length is an important parameter because it closely defines the quality of the received signal $[8,14]$. A longer filter length implies superior BER performance, however comes at the cost of additional computational complexity.

The symbols are then convolved with the pulse shaping filters are then used to generate the signal $s(t)$ as follows [9]:

$$
s(t)=a_{I} \otimes p(t)-a_{Q} \otimes p^{\prime}(t)
$$

where $a_{I}$ and $a_{Q}$ are the real and imaginary components of the complex QAM symbols and $\otimes$ denotes time-domain convolution. The sampling frequency is locked at $f_{s}=R_{s} n_{s s}$. The board used is a National Instruments universal software peripheral reconfigurable input-output (NI USRP-RIO) 2953 which has a Xilinx Kintex 7 (410T) field programmable gate array on-board. After the signal is generated in the NI USRPRIO, the signal is passed to an Ettus Research LFTX digital-toanalogue converter (DAC). The signal is divided into a frame structure each consisting of 1,000 bits in order to keep the frame size small. The first frame of every 100 frames is used entirely for synchronisation and phase/amplitude correction in the common phase error (CPE) correction stage of the block diagram. The signal is output with $0.5 \mathrm{~V}$ swing to a custom light-emitting diode (LED) driver, which consists of a Texas Instruments THS3202 amplifier set to amplify the signal by four times to $2 \mathrm{~V}$ and a bias tee that sets the DC current to $500 \mathrm{~mA}$, which is the centre of the linear region of the LED used. As in [9], the LED is an Osram Golden Dragon, with $2 \mathrm{MHz}$ bandwidth. Unlike [9], where the link distance was $0.1 \mathrm{~m}$, here the distance is increased to $0.5 \mathrm{~m}$ because a larger photodiode is used. The transmit power of the LED was $1.5 \mathrm{~mW}$ and any ambient light effects were limited by conducting the experiment in a dark laboratory.

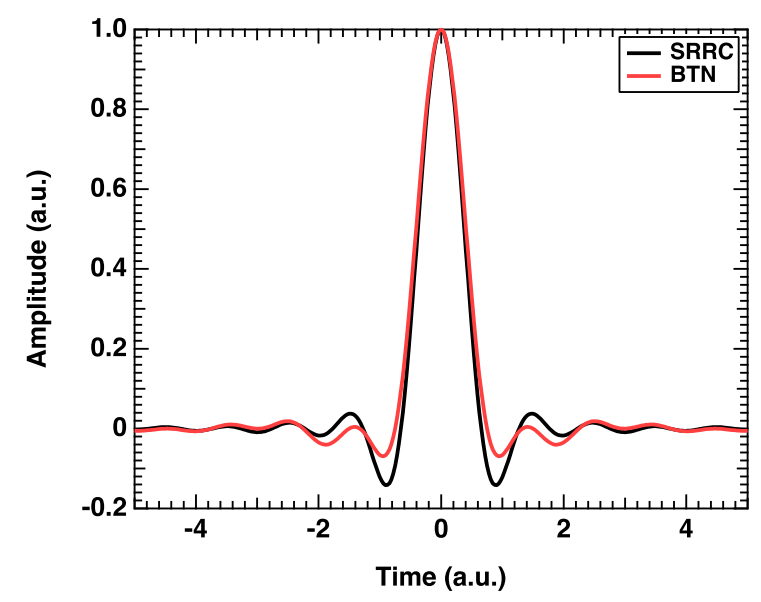

Fig. 2. The two candidate basis function pulse shapes

In this work, a ThorLabs PDA36A2 photodiode with $75.4 \mathrm{~mm}^{2}$ area and an in-built transimpedance amplifier is used, thus enabling an improved link distance whilst maintaining the same signal level as in [9]. Next, a second Texas Instruments THS3202 amplifier is used to set the received signal level to $0.5 \mathrm{~V}_{\mathrm{AC}}$ which properly utilises the full quantisation range of the Ettus Research LFRX analogue-to-digital 


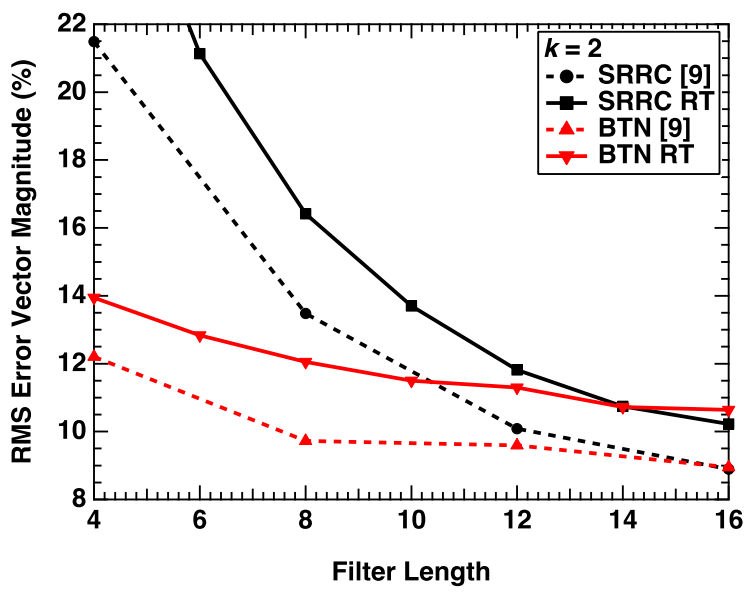

Fig. 3. EVM performance of 4-QAM for both the BTN and SRRC in RT compared with previous literature [9]

converter (ADC). The receiver is a NI USRP-RIO 2943 with the same Kintex 7 FPGA running independently from the transmitter. At the digitisation stage, the signal is sampled at $f_{s}$ and synchronised through standard methods. Two different methods are used for detection; (i) the conventional method, where time-reversed matched filters are used to demodulate the signal, where the in-phase and quadrature matched filters are given by $p(-t)$ and $p^{\prime}(-t)$, respectively, and (ii) the method proposed in $[4,9]$ where a local oscillator and 10th order lowpass filter with cut-off frequency $R_{s}$ is used to demodulate the signal. The second method was demonstrated to alleviate the dependence on $L_{s}$ due to the use of a full-Nyquist pulse and the absence of long filters at the receiver.

Next, CPE is removed before constellation de-mapping and estimation of the transmitted binary sequence vector $\mathbf{E}_{\mathbf{s}}$. We investigate the comparative error vector magnitude (EVM) performance of the SRRC and BTN pulse shapes for 4-, 16and 64-QAM. We analyse the impact of varying the filter length of each pulse shape on the link in RT on the EVM and compare it with results reported in an offline system in previous literature [9]. The filter lengths $L_{s}$ tested span from 4 symbols up to 16 symbols in steps of 2 . For the entirety of this work, $\beta=0.1$, which forces more energy into the side-lobes, and is the value commonly found in the literature [1,15-17].

\section{RESULTS}

The EVM results for the two candidate pulse shapes are illustrated as a function of filter length in Fig. 3. It can immediately be observed that the trend of the two datasets are comparable, i.e. the SRRC-based CAP offers significant deviation of EVM over the range of $L_{S}$ tested. Starting with $L_{s}=4$, the measured EVM is greater than $20 \%$ for both the previous report and this experiment, decreasing to an EVM of $\sim 10-12 \%$ for $L_{s}=16$.

The key difference between the two datasets is that the RT result measured here consistently offers $1-2 \%$ inferior EVM performance. The reason for this is attributed to the imperfect synchronisation of the received signal. In [9] the signal is synchronised offline in MATLAB through cross-correlation with a perfect version of the transmitted symbol, then both the perfect and received versions of the signal are oversampled to 10 times the original sampling frequency before fine-offset adjustment and down-sampling to the original rate. This provides a very close synchronisation between the two, which results in the highest quality demodulation available. This technique, which would be extremely costly in terms of signal processing computational complexity and the latency it would introduce, is not available in RT. Therefore, Schmidl and Cox synchronisation is used, which effectively provides a similar functionality by first performing a loose synchronisation followed by a fine adjustment, but without the large oversampling factor [18].

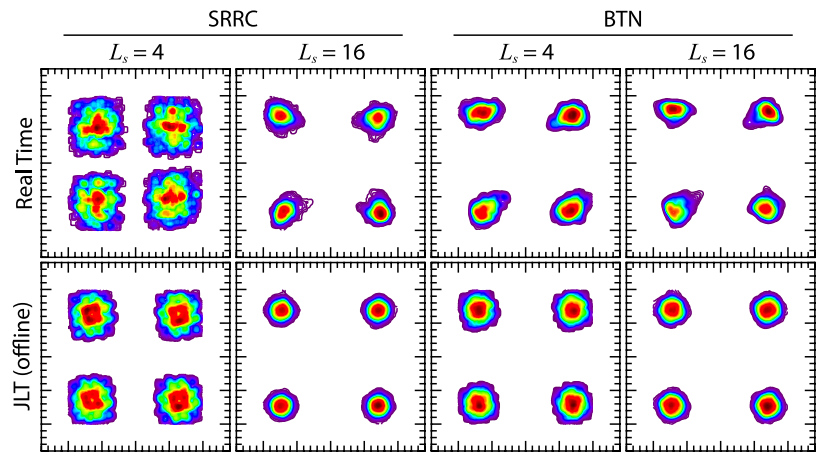

Fig. 4. Constellations for 4-QAM

The inexact synchronisation leads to a small reduction of EVM performance. This is reflected by the shape of the received constellation (see Fig. 4, which shows there are amplitude variations between received symbols as the shape is no longer approximately Gaussian.

The BTN-based CAP shows a similar pattern, maintaining the $\sim 1-2 \%$ EVM penalty in the RT system. Interestingly, as reported in [9], the BTN pulse offers significantly improved performance at short filter lengths, reducing the EVM from $>20 \%$ to $\sim 12 \%$ and $14 \%$ for the previous work [9] and this system, respectively. The reason for this is due to the faster damping of the BTN in comparison to the SRRC and the fact that midpoint sampling can be directly performed at the receiver, instead of a matched filter, as the BTN is a fullNyquist pulse shape. Therefore, the penalty is significantly reduced, and a significantly improved constellation can be observed in Fig. 4. The EVM settles at similar values to the SRRC for both cases at longer filter lengths. For $L_{s}>16$, no further improvement is observed and the EVM values are simply maintained.

Similar trends are observed for higher orders of QAM, as illustrated in Fig. 5, which shows the same EVM profile for 16-QAM. Similar trends are observed such that the BTN-based CAP significantly outperforms its SRRC-based equivalent at low filter lengths, while all pulses converge to similar EVM performances at long filter lengths. This time, the EVM penalty is $\sim 1-3 \%$, which is slightly increased, and the reason for this is the higher SNR requirement for 16QAM in comparison to 4-QAM. Constellations are illustrated in Fig. 6 for $L_{s}=4$ and 16 for all cases. A similar trend was also observed for the 64-QAM performance, demonstrating the generality of the system. Unfortunately, 64-QAM data was not 
obtained in [9], so due to space considerations, the 64-QAM data is not shown.

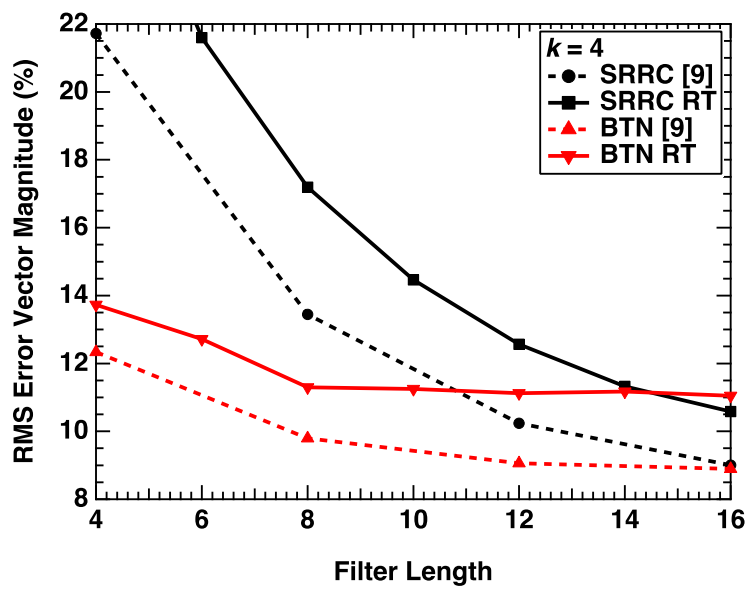

Fig. 5. EVM performance of 16-QAM for both the BTN and SRRC in RT compared with previous literature [9]

\section{Conclusion}

In this paper we have demonstrated RT CAP modulation in VLC for the first time, using both SRRC and BTN pulses. We show that the BTN pulse comprehensively outperforms the SRRC due to the fact that the pulse damps faster and hence, can tolerate ISI even at short filter spans, unlike the SRRC. We have confirmed the generality of the BTN-CAP performance improvement across modulation orders. The RT penalty is an additional 3\% EVM in the worst case over offline processing and arrives as a result of imperfect synchronisation of the signal at the receiver.

\section{REFERENCES}

[1] F. M. Wu, C. T. Lin, C. C. Wei, C. W. Chen, Z. Y. Chen, H. T. Huang, and S. Chi, "Performance comparison of OFDM Signal and CAP signal over high capacity RGB-LED-based WDM visible light communication," IEEE Photonics Journal, vol. 5, no. 4, pp. $7901507-$ $7901507,2013$.

[2] Y. G. Wang, L. Tao, X. X. Huang, J. Y. Shi, and N. Chi, "8-Gb/s RGBY LED-based WDM VLC system employing high-order CAP modulation and hybrid post equalizer," IEEE Photonics Journal, vol. 7, no. 6, pp. $1-7,2015$

[3] S. Liang, Z. Jiang, L. Qiao, X. Lu, and N. Chi, "Faster-than-Nyquist pre-coded CAP modulation visible light communication system based on nonlinear weighted look-up table pre-distortion," IEEE Photonics Journal, 2018

[4] J. L. Wei, C. Sanchez, and E. Giacoumidis, "Fair comparison of complexity between a multi-band CAP and DMT for data center interconnects," Optics Letters, vol. 42, no. 19, pp. 3860-3863, 2017.

[5] R. Schmogrow, R. Bouziane, M. Meyer, P. A. Milder, P. C. Schindler, R. I. Killey, P. Bayvel, C. Koos, W. Freude, and J. Leuthold, "Real-time OFDM or nyquist pulse generation - which performs better with limited resources?" Optics Express, vol. 20, no. 26, pp. B543-B551, 2012.

[6] P. A. Haigh and I. Darwazeh, "Demonstration of reduced complexity multi-band CAP modulation using Xia-pulses in visible light communications," in 2018 Optical Fiber Communications Conference and Exposition (OFC). IEEE, Conference Proceedings, pp. 1-3.

[7] D. Falconer, "Carrierless am/pm," Bell Laboratories Technical Memorandum, Tech. Rep, 1975.

[8] P. Chvojka, K. Werfli, S. Zvanovec, P. A. Haigh, V. H. Vacek, P. Dvorak, P. Pesek, and Z. Ghassemlooy, "On the $m$-CAP performance with different pulse shaping filters parameters for visible light communications," IEEE Photonics Journal, vol. 9, no. 5, pp. 1-12, 2017.

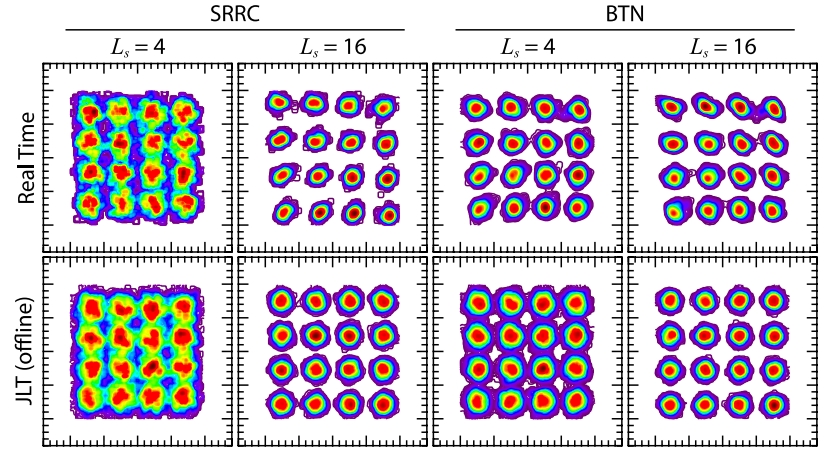

Fig. 6. Constellations for 16-QAM

[9] P. A. Haigh, P. Chvojka, S. Zvánovec, Z. Ghassemlooy, and I. Darwazeh, "Analysis of Nyquist pulse shapes for carrierless amplitude and phase modulation in visible light communications," IEEE/OSA Journal of Lightwave Technology, vol. 36, no. 20, pp. 5023-5029, 2018.

[10] Z. Li, C. Zhang, D. Sun, H. Yang, and J. Song, "A real-time highspeed visible light communication system based on RGB-LEDs," in 2017 IEEE International Symposium on Broadband Multimedia Systems and Broadcasting (BMSB), Conference Proceedings, pp. 1-4.

[11] F. Wang, J. Yin, J. Shi, N. Chi, L. Lu, A. Xu, J. Xiong, and Z. Ma, "Real-time bi-directional $800 \mathrm{Mb} / \mathrm{s}$ visible light communication system utilizing commercial LEDs," in Asia Communications and Photonics Conference 2016. OSA, p. AF3A.5.

[12] Y. Mao, X. Jin, W. Liu, C. Gong, and Z. Xu, "Demonstration of realtime cap transceivers with hybrid digital equalization for visible light communication," in Asia Communications and Photonics Conference. Optical Society of America, 2017, p. M1G.4. [Online]. Available: http://www.osapublishing.org/abstract.cfm?URI=ACPC-2017-M1G.4

[13] Y. Mao, X. Jin, W. Pan, W. Liu, M. Jin, C. Gong, and Z. Xu, "Realtime investigation of cap transceivers with hybrid digital equalization for visible light communication," Optics express, vol. 27, no. 7, pp. 9382-9393, 2019.

[14] P. Chvojka, P. A. Haigh, S. Zvanovec, P. Pesek, and Z. Ghassemlooy, "Evaluation of multi-band carrier-less amplitude and phase modulation performance for VLC under various pulse shaping filter parameters," in OPTICS, pp. 25-31.

[15] M. Tavan, E. Agrell, and J. Karout, "Bandlimited intensity modulation," IEEE Transactions on Communications, vol. 60, no. 11, pp. 3429-3439, 2012.

[16] P. A. Haigh, A. Burton, K. Werfli, H. L. Minh, E. Bentley, P. Chvojka, W. O. Popoola, I. Papakonstantinou, and S. Zvanovec, "A multiCAP visible-light communications system with $4.85-\mathrm{b} / \mathrm{s} / \mathrm{Hz}$ spectral efficiency," IEEE Journal on Selected Areas in Communications, vol. 33, no. 9, pp. 1771-1779, 2015.

[17] N. C. Beaulieu, C. C. Tan, and M. O. Damen, "A "better than" Nyquist pulse," IEEE Communications Letters, vol. 5, no. 9, pp. 367-368, 2001.

[18] T. M. Schmidl and D. C. Cox, "Robust frequency and timing synchronization for OFDM," IEEE transactions on communications, vol. 45, no. 12 , pp. 1613-1621, 1997. 